\title{
INTENSITIES OF 4f-4f TRANSITIONS IN GLASS MATERIALS
}

\section{Oscar L. Malta*}

Departamento de Química Fundamental, Centro de Ciências Exatas e da Natureza, Universidade Federal de Pernambuco, 50670-901 Recife - PE

\section{Luís D. Carlos}

Departamento de Física, Centro de Investigação em Materiais Cerâmicos e Compósitos, Universidade de Aveiro, 3810-193 Aveiro, Portugal

Recebido em 2/12/02; aceito em 10/4/03

\begin{abstract}
In this article we review some of the basic aspects of rare earth spectroscopy applied to vitreous materials. The characteristics of the intra-atomic free ion and ligand field interactions, as well as the formalisms of the forced electric dipole and dynamic coupling mechanisms of $4 \mathrm{f}-4 \mathrm{f}$ intensities, are outlined. The contribution of the later mechanism to the $4 \mathrm{f}-4 \mathrm{f}$ intensities is critically discussed, a point that has been commonly overlooked in the literature of rare earth doped glasses. The observed correlation between the empirical intensity parameter $\Omega_{2}$ and the covalence of the ion first coordination shell is discussed accordingly to the theoretical predictions.
\end{abstract}

Keywords: rare earth ions; photoluminescence spectroscopy; $4 \mathrm{f}-4 \mathrm{f}$ intensities.

\section{INTRODUCTION}

Glass materials containing trivalent rare earth ions have been widely studied due to their potential applications as optical devices (lasers and fibers for optical amplifiers, among others) or as the active component in photonics ${ }^{1-3}$. For instance, high-power terawatt lasers ( $10^{12} \mathrm{~W}$ is equivalent to 1000 joules released in a nanosecond pulse) incorporating $\mathrm{Nd}^{3+}$ ions are currently used for thermonuclear fusion ${ }^{1}$. Recently there has been also a great deal of interest in the conversion of infrared into visible light by energy up-conversion, due to the potential application in photonics, including optical data storage, lasers, sensors, and optical displays ${ }^{5,6}$. The rare-earth doped glasses have indeed a great advantage over crystalline systems since generally they can be easily prepared with high optical quality and in a large variety of chemical compositions. They can be used either in large bulk devices or in optical fiber waveguides to confine the pumping light with a high density over a long interaction length.

The optical properties of these materials are directly related to the $4 \mathrm{f}-4 \mathrm{f}$ transitions in a $4 \mathrm{f}^{\mathrm{N}}$ electronic configuration. In our days, the theoretical background for the rationalization of these intraconfigurational transitions, both radiative and non-radiative, is well established ${ }^{7-17}$. The standard Judd-Ofelt theory ${ }^{7,8}$ has been used to evaluate absorption and emission cross-sections in the great majority of the works on these rare earth doped glasses. The socalled intensity parameters $\Omega_{\lambda}(\lambda=2,4$ and 6$)$ have been used to give information on covalency, quality and mechanical properties of the medium ${ }^{1}$. Nevertheless, a certain number of problems still exist, related to the application of the theory and to the interpretation of results obtained from it, particularly in the case of glasses, as one may note from the literature. Among these problems we may emphasize the systematic neglect of the dynamic coupling mechanism contributing to the $4 \mathrm{f}-4 \mathrm{f}$ intensities ${ }^{10-14,16}$.

We wish here to outline the main aspects of the aforementioned theoretical background and to discuss briefly some rather problematic

*e-mail: omlm@npd.ufpe.br points concerning the use of the theory and the interpretation of results.

\section{SOME CHARACTERISTICS OF THE RARE EARTH IONS}

\section{The Hamiltonian for the free ion}

The Hamiltonian, $\mathrm{H}_{\mathrm{F}}$, for the rare earth free ion is composed by one part due to the central field, $\mathrm{H}_{0}$, and by several other interactions, which are generally treated as perturbations. Among these interactions the interelectronic repulsion, $\mathrm{H}_{\mathrm{c}}$, and the spin-orbit interaction, $\mathrm{H}_{\mathrm{so}}$, are the most relevant.

$\mathrm{H}_{\mathrm{FI}}=\mathrm{H}_{0}+\mathrm{H}_{\mathrm{c}}+\mathrm{H}_{\mathrm{so}}$ $\mathrm{H}_{\mathrm{s}}$

The magnitudes of these interactions follow the order $\mathrm{H}_{0}>\mathrm{H}_{\mathrm{c}}>$

In the diagonalization procedure of the Hamiltonian $\mathrm{H}_{\mathrm{FI}}$ the spinspin, spin-other-orbit and orbit-orbit interactions are in general of much less importance. Thus, in a first step, the eigenfunctions of $\mathrm{H}_{\mathrm{FI}}$ may be constructed from the eigenfunctions of the angular momentum operators $\mathbf{L}^{2}, \mathbf{S}^{2}, \mathbf{J}^{2}$ and $\mathbf{J}_{\mathrm{Z}}$. $\mathbf{L}$ is the total orbital angular momentum, $\mathbf{S}$ the total spin angular momentum, $\mathbf{J}$ the total angular momentum, $\mathbf{J}=\mathbf{L}+\mathbf{S}, \mathbf{J}_{\mathrm{Z}}$ the $\mathrm{z}$ component of $\mathbf{J}$, with $\mathbf{L}=\Sigma \ell_{i}$ and $\mathbf{S}=\Sigma s_{i}, \ell$ and $s$ being monoelectronic orbital and spin angular momentum, respectively. The quantum number $\mathrm{J}(\mathrm{MJ}=-\mathrm{J},-\mathrm{J}+1, \ldots,+\mathrm{J})$ must satisfy the condition $|\mathrm{L}-\mathrm{S}| \leq \mathrm{J} \leq \mathrm{L}+\mathrm{S}$. For rare earth ions, an adequate scheme to represent a basis of eigenfunctions is the wellknown L-S coupling scheme $\left|\left(4 \mathrm{f}^{\mathrm{N}}\right) \alpha \mathrm{SLJM}_{\mathrm{J}}\right\rangle$. This notation implies that these eigenstates are pure $4 \mathrm{f}^{\mathrm{N}}$ states, or, in other words, no configuration interaction (CI) via $\mathrm{H}_{\mathrm{FI}}$ is taken into account. CI effects, for each given case, have been considered without increasing the dimension of the matrix $\left[\mathrm{H}_{\mathrm{FI}}\right]$ through the use of effective operators acting within the $4 \mathrm{f}^{\mathrm{N}}$ configuration.

In the construction of the eigenstates $\left|\left(4 \mathrm{f}^{\mathrm{N}}\right) \alpha \mathrm{SLJM}_{\mathrm{J}}\right\rangle$ one frequently finds that they are not unambiguously defined by the quantum numbers $\mathrm{S}, \mathrm{L}, \mathrm{J}$ and $\mathrm{M}_{\mathrm{J}}$. This problem can be solved by the 
use of group theory. Racah ${ }^{18}$ has demonstrated that the irreducible representations of certain sub-groups of the GL $(4 \ell+2)$ continuous group may be used as quantum numbers to classify these eigenstates. This is due to the fact that they form bases for these irreducible representations. Thus, in the above scheme a represents the set of additional quantum numbers necessary to specify the eigenstate. A common procedure in the literature has been to diagonalize the Hamiltonian $\mathrm{H}_{\mathrm{FI}}$ on a basis formed by the above eigenstates. The usual form of $\mathrm{H}_{\mathrm{FI}}$ is ${ }^{19,20}$ :

$$
H_{F I}=E_{b}\left(4 f^{N}\right)+\sum_{k=0,1,2,3} E^{k} e_{k}+\zeta_{4 f} \sum_{i} \vec{\ell}_{i} \cdot \vec{s}_{i}+\mathrm{CI}
$$

where $E_{b}$ is the energy of the baricenter of the $4 \mathrm{f}^{\mathrm{N}}$ configuration (eigenvalue of the central field Hamiltonian). The second and third terms in the right-hand-side of this expression correspond to the interelectronic repulsion and spin-orbit interaction, respectively. The last term contains configuration interaction effects. Matrix elements of the components in equation (2) are evaluated by irreducible tensor operator techniques ${ }^{19-22}$. The radial quantities in equation (2), $\mathrm{E}_{\mathrm{k}}$ and $\zeta_{4 f}$, the Racah and spin-orbit parameters, respectively, may in principle be calculated from ab initio methods (the $\mathrm{E}^{0}$ parameter can be absorbed by $\mathrm{E}_{\mathrm{b}}$ ). However they are commonly treated as adjustable parameters, for which the input data are the experimentally observed energies of the baricenters of the $\mathrm{J}$ manifolds. The great advantage of this latter procedure is that, after diagonalization, one gets usually much more reliable free ion wavefunctions, what is essential to the evaluation of properties of rare earth ion doped materials.

\section{The eigenfunctions in the intermediate coupling}

Taking into account the fact that the interelectronic repulsion is not diagonal in the quantum number $\alpha$ and that the spin-orbit interaction is not diagonal in the quantum numbers $\alpha, \mathrm{S}$ and $\mathrm{L}$, these latter are no longer good quantum numbers. As a consequence, after diagonalization of $\mathrm{H}_{\mathrm{FI}}$, the eigenstates will be given by a linear combination of the states $\left|\left(4 \mathrm{f}^{\mathrm{N}}\right) \alpha \mathrm{SLJM}_{\mathrm{J}}\right\rangle$, that is,

$$
\left|\left(4 \mathrm{f}^{\mathrm{N}}\right) \psi \mathrm{JM}_{\mathrm{J}}\right\rangle=\sum_{\alpha \mathrm{S} \mathrm{L}} \mathrm{C}(\alpha \mathrm{SL})\left|\left(4 \mathrm{f}^{\mathrm{N}}\right) \alpha \mathrm{SLJM}_{\mathrm{J}}\right\rangle
$$

in which the $\mathrm{C}(\alpha \mathrm{SL})$ coefficients of the linear combination satisfies the condition

$$
\sum_{\alpha \mathrm{S} L}|\mathrm{C}(\alpha \mathrm{SL})|^{2}=1
$$

Equation (3) expresses the so-called intermediate coupling scheme. The eigenstates given in this scheme, as mentioned before, are essential to describe the behavior of rare earth ions. A good example is the case of transitions between multiplets of different multiplicities, which otherwise cannot be described. A typical case is illustrated by the transitions between the ${ }^{5} \mathrm{D}_{\mathrm{J}}$ and ${ }^{7} \mathrm{~F}_{\mathrm{J}}$ multiplets of the $\mathrm{Eu}^{3+}$ ion. Labeling a multiplet by the usual notation ${ }^{2 \mathrm{~S}+1} \mathrm{~L}_{\mathrm{J}}$ is a mere indication of the dominant component in the summation in equation (3). An interesting and useful aspect is that, since a rather weakly chemical environment affects the $4 \mathrm{f}$ orbitals, for each rare earth ion the eigenstates in the intermediate coupling scheme are essentially the same for different environments.

\section{THE LIGAND FIELD}

\section{The usual form of the ligand field Hamiltonian}

Although weak, the interaction between $4 \mathrm{f}$ electrons and the chemical environment is responsible for the most interesting spectroscopic features of rare earth ions. The non-spherical even parity part of this interaction, responsible for the Stark splitting of $4 \mathrm{f}$ levels, is commonly written as:

$\mathrm{H}_{\mathrm{LF}}=\sum_{\mathrm{k}, \mathrm{q}, \mathrm{i}} \mathrm{B}_{\mathrm{q}}^{\mathrm{k}} \mathrm{C}_{\mathrm{q}}^{(\mathrm{k})}(\mathrm{i})$

where the $\mathrm{B}_{\mathrm{q}}^{\mathrm{k}}$ 's $(\mathrm{k}=2,4$ and 6$)$ are the so-called ligand field parameters of even rank and $\mathbf{C}^{(\mathrm{k})}$ is a Racah tensor operator of rank $\mathrm{k}^{19-22}$. The values of $\mathrm{k}$ are restricted by parity and triangularity rules for $\mathrm{f}$ orbitals ${ }^{19,20}$. The allowed values of $q$ depend on the symmetry of the ligand field around the rare earth ion, and in this expression the index i runs over the $4 \mathrm{f}$ electrons.

The Hamiltonian $\mathrm{H}_{\mathrm{LF}}$ as given by equation (5) is a one-particle operator. A relevant point here is that the form of equation (5) has a more general character than it might be supposed, in the sense that all one particle ligand field models lead to this form of $\mathrm{H}_{\mathrm{LF}}$. Despite the fact that the $\mathrm{B}_{\mathrm{q}}^{\mathrm{k}}$ 's, for a given point symmetry, can be calculated from theoretical models, it has been a common practice to treat them also as adjustable parameters called phenomenological or experimental $\mathrm{B}_{\mathrm{q}}^{\mathrm{k}}$ 's. As for the case of the free ion radial parameters in equation (2), the input data are the observed energies of the $4 \mathrm{f}$ levels under the action of the ligand field. The total Hamiltonian to be diagonalized is now

$\mathrm{H}=\mathrm{H}_{\mathrm{FI}}+\mathrm{H}_{\mathrm{LF}}$

The ligand field interaction is also of fundamental importance for the case of $4 \mathrm{f}-4 \mathrm{f}$ transition intensities. These transitions are in principle electric dipole forbidden by Laporte's rule. However, provided that the site occupied by the rare earth ion does not present a center of inversion, Laporte's rule is relaxed due to odd parity terms in the ligand field Hamiltonian. The more general form of $\mathrm{H}_{\mathrm{LF}}$ is actually

$\mathrm{H}_{\mathrm{LF}}=\mathrm{H}_{\mathrm{LF}}($ even $)+\mathrm{H}_{\mathrm{LF}}($ odd $)$

It is important to note that if the diagonalization of the total Hamiltonian $\mathrm{H}$ in equation (6) is restricted to a basis formed by the states $\left|\left(4 \mathrm{f}^{\mathrm{N}}\right) \alpha \mathrm{SLJM}_{\mathrm{J}}\right\rangle$, due to parity selection rules, the component $\mathrm{H}_{\mathrm{LF}}$ (odd) will have no effect on the final results. This odd component is generally expressed as:

$\mathrm{H}_{\mathrm{LF}}(\mathrm{odd})=\sum_{\mathrm{t}, \mathrm{p}, \mathrm{i}} \gamma_{\mathrm{p}}^{\mathrm{t}} \mathrm{r}_{\mathrm{i}}^{\mathrm{t}} \mathrm{C}_{\mathrm{p}}^{(\mathrm{t})}(\mathrm{i})$

where $r_{i}$ is the radial coordinate of the $i$-th electron, $\gamma_{p}^{t}$ 's $(t=1,3,5$ and 7) are the so-called odd rank ligand field parameters, and $\mathbf{C}^{(t)}$ is a Racah tensor operator of rank $t$. The values of $t$ are restricted by parity and triangularity rules involving $\mathrm{f}, \mathrm{d}$ and $\mathrm{g}$ orbitals $\mathrm{s}^{7,8}$. Now the index i runs, in principle, over all electrons of the rare earth ion. As for the values of $q$ in equation (5), the allowed values of $p$ depend on the symmetry around the rare earth ion. In the case of intensities the role of $\mathrm{H}_{\mathrm{LF}}$ (odd) is to connect (mix) states belonging to electronic configurations of opposite parity. It follows that transitions between 4f levels become partially electric dipole allowed.

\section{The ligand field and symmetry}

As previously mentioned, the values of $\mathrm{q}$ and $\mathrm{p}$ in equations (5) and (8), respectively, are restricted by the symmetry of the site occupied by the rare earth ion. Thus, for example, in a $\mathrm{C}_{4 \mathrm{~V}}$ symmetry the allowed values are: $\mathrm{k}=2, \mathrm{q}=0 ; \mathrm{k}=4, \mathrm{q}=0, \pm 4 ; \mathrm{k}=6, \mathrm{q}=0, \pm$ $4 ; \mathrm{t}=1, \mathrm{p}=0 ; \mathrm{t}=3, \mathrm{p}=0 ; \mathrm{t}=5, \mathrm{p}=0, \pm 4 ; \mathrm{t}=7, \mathrm{p}=0, \pm 4$. This is 
a consequence of the fact that the ligand field parameters are actually a summation over the individual contributions from the surrounding atoms. Each individual contribution behaves as the spherical harmonics and the summation vanishes in a given symmetry for certain values of $\mathrm{q}$ and $\mathrm{p}$. A detailed work on this subject may be found elsewhere $\mathrm{e}^{23,24}$.

One of the consequences of the action of $\mathrm{H}_{\mathrm{LF}}$ (even) is that $\mathrm{J}$ is no longer a good quantum number. This produces the so-called J-mixing effect (a rather small effect due to the weak interaction between the $4 \mathrm{f}$ orbitals and the chemical environment), and as a result of the diagonalization of the total Hamiltonian $\mathrm{H}$ in equation (6) the final eigenstates have the general form:

$$
\left|\left(4 \mathrm{f}^{\mathrm{N}}\right) \Gamma\right\rangle=\sum_{\alpha, \mathrm{S}, \mathrm{L}, \mathrm{J}, \mathrm{M}_{\mathrm{J}}} \mathrm{A}\left(\alpha, \mathrm{S}, \mathrm{L}, \mathrm{J}, \mathrm{M}_{\mathrm{J}} ; \Gamma\right)\left|\left(4 \mathrm{f}^{\mathrm{N}}\right) \alpha \mathrm{SLJM}_{\mathrm{J}}\right\rangle
$$

with the condition

$$
\sum_{\alpha, \mathrm{S}, \mathrm{L}, \mathrm{J}, \mathrm{M}_{\mathrm{J}}}\left|\mathrm{A}\left(\alpha, \mathrm{S}, \mathrm{L}, \mathrm{J}, \mathrm{M}_{\mathrm{J}} ; \Gamma\right)\right|^{2}=1
$$

Each eigenstate given by equation (9) is now labeled by an irreducible representation, $\Gamma$, of the symmetry point group.

In the case of glasses, since there is a variety of different sites that can be occupied by the rare earth ion, we cannot talk about a well-defined set of ligand field parameters. The Stark splitting is in general not well determined in this case, what makes it difficult to define even an average set of ligand field parameters.

In Figure 1 a schematic representation of the intra-atomic and ligand field interactions previously discussed is presented.

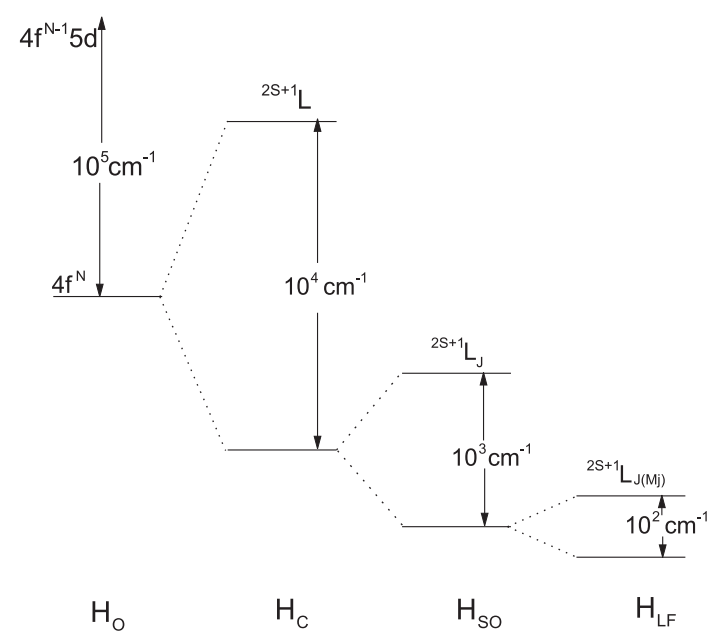

Figure 1. Schematic representation and order of magnitude of the effects of the intra-atomic and ligand field interactions acting on a $4 f^{N}$ configuration

\section{F-4F INTENSITIES}

\section{Mechanisms of $\mathbf{4 f}-\mathbf{4 f}$ intensities}

The characteristic absorption and emission spectra of rare earth compounds in the visible, near ultraviolet and near infrared is attributed to transitions between $4 \mathrm{f}$ levels, as they present sharp lines, mainly at low temperature, with oscillator strengths typically in the order of 10 ${ }^{6}$. These transitions are forbidden to first order by electric dipoles, but are allowed by the electric quadrupole, vibronic, magnetic dipole and forced electric dipole mechanisms. It has been noticed, since more than fifty years ago, that among these only the magnetic dipole and forced electric dipole mechanisms could account for the observed intensities ${ }^{25}$. The magnetic dipole character of the ${ }^{5} \mathrm{D}_{0} \rightarrow{ }^{7} \mathrm{~F}_{1}$ transition of the $\mathrm{Eu}^{3+}$ ion was demonstrated in 1939 by Deutschbein ${ }^{26}$. The coefficient of spontaneous emission between two manifolds $\mathrm{J}$ and $\mathrm{J}^{\prime}$, due to the magnetic dipole mechanism, is given by:

$$
\mathrm{A}_{\mathrm{J}, \mathrm{J}^{\prime}}=\frac{4}{3} \frac{\mathrm{e}^{2} \omega^{3}}{\hbar \mathrm{c}^{3}} \mathrm{n}^{3} \mathrm{~S}_{\mathrm{md}}
$$

where the magnetic dipole line strength $\mathrm{S}_{\mathrm{md}}$ (in units of $\mathrm{e}^{2}$, where $\mathrm{e}$ is the electronic charge, $\mathrm{e}=-4.8 \times 10^{-10}$ e.s.u. $)$, is

$$
\mathrm{S}_{\mathrm{md}}=\frac{\hbar^{2}}{4 \mathrm{~m}_{\mathrm{e}} \mathrm{c}^{2}}\left|\left\langle\left(4 \mathrm{f}^{\mathrm{N}}\right) \psi^{\prime} \mathrm{J}^{\prime}\|\mathrm{L}+2 \mathrm{~S}\|\left(4 \mathrm{f}^{\mathrm{N}}\right) \psi \mathrm{J}\right\rangle\right|^{2} \frac{1}{2 \mathrm{~J}+1}
$$

In the previous equations, $w$ is the angular frequency of the $\mathrm{J} \rightarrow \mathrm{J}^{\prime}$ transition $\left(\omega=2 \pi c \sigma, \sigma\right.$ being the transition energy in $\left.\mathrm{cm}^{-1}\right), \mathrm{n}$ is the refraction index of the medium and the angular momentum operators $\mathrm{L}$ and $\mathrm{S}$ are in units of $\hbar$. The eigenstates in equation (12) are calculated using the intermediate coupling scheme. The magnetic dipole mechanism cannot account for most of the $4 \mathrm{f}-4 \mathrm{f}$ transitions in the rare earth series, not only because the predicted oscillator strengths are in general smaller than $10^{-6}$ but also due to the restrictive selection rules on the $\mathrm{J}$ quantum number $(\Delta \mathrm{J}=0, \pm 1)$, as far as $\mathrm{J}$ is considered a good quantum number.

$\mathrm{Judd}^{7}$ and Ofelt ${ }^{8}$ treated the forced electric dipole mechanism in detail for the first time in 1962 through the powerful technique of irreducible tensor operators ${ }^{19-22}$. Two years later Jorgensen and Judd ${ }^{27}$ studied the influence of an additional $4 \mathrm{f}-4 \mathrm{f}$ mechanism. It was originally referred to as the pseudoquadrupolar mechanism, due to inhomogeneities of the dielectric constant. The authors proposed that it could be as operative as, or, for some transitions, even more relevant than the forced electric dipole mechanism. These two processes will be briefly described in the next two sub-sections.

\section{The Judd-Ofelt theory}

The electric dipole strength, $\mathrm{S}_{\mathrm{ed}}$ (in units of $\mathrm{e}^{2}$ ), of a transition between two states $\phi$ and $\phi^{\prime}$ is given by

$$
\mathrm{S}_{\mathrm{ed}}=\left|\left\langle\phi^{\prime}\left|\sum_{\mathrm{i}} \overrightarrow{\mathrm{r}}_{\mathrm{i}}\right| \phi\right\rangle\right|^{2}
$$

If the states $\phi$ and $\phi^{\prime}$ are pure $4 \mathrm{f}^{\mathrm{N}}$ states, as those given by equations (3) and (9), then by the parity selection rule (Laporte's rule) the dipole strength $S_{\text {ed }}$ is identically null. However, provided that there is no center of inversion in the site occupied by the rare earth ion, this selection rule is relaxed by the odd component of the ligand field Hamiltonian, $\mathrm{H}_{\mathrm{LF}}$ (odd), which mixes states of electronic configurations with opposite parity. Thus, since $\mathrm{H}_{\mathrm{LF}}$ (odd) is a one particle operator, the configurations that can be mixed with the ground $4 \mathrm{f}^{\mathrm{N}}$ configuration are those of the type $4 \mathrm{f}^{\mathrm{N}-1} \mathrm{nd}, 4 \mathrm{f}^{\mathrm{N}-1} \mathrm{ng}(\mathrm{n} \geq 5)$ and $\mathrm{n}^{\prime} \mathrm{d}^{4 \mathrm{~d}+1} 4 \mathrm{f}^{\mathrm{N}+1}\left(\mathrm{~d}=2, \mathrm{n}^{\prime}=3\right.$ and 4 , corresponding to core excitations). In the standard Judd-Ofelt theory the initial step is to consider this mixing by means of perturbation theory up to first order in the wavefunctions. If we take the perturbation on the eigenstates given by equation (9), then we may write:

$|\phi\rangle=\left|\left(4 \mathrm{f}^{\mathrm{N}}\right) \Gamma\right\rangle+\sum_{\mathrm{B} \beta} \frac{\left\langle\mathrm{B} \beta\left|\mathrm{H}_{\mathrm{LF}}(\mathrm{odd})\right|\left(4 \mathrm{f}^{\mathrm{N}}\right) \Gamma\right\rangle}{\mathrm{E}(\Gamma)-\mathrm{E}(\mathrm{B} \beta)}|\mathrm{B} \beta\rangle$

where B designates an excited configuration of opposite parity and $\beta$ its states. The state $\left|\phi^{\prime}\right\rangle$ has a similar expression. The matrix element in equation (13), abbreviated as $\vec{\mu}_{\text {ed }}$, is consequently given by: 
$\vec{\mu}_{\mathrm{ed}}=\sum_{\mathrm{BB}}\left[\frac{\left\langle\left(4 \mathrm{f}^{\mathrm{N}}\right) \Gamma^{\prime}\left|\sum_{\mathrm{i}} \overrightarrow{\mathrm{r}}_{\mathrm{i}}\right| \mathrm{B} \beta\right\rangle\langle\mathrm{B} \beta| \mathrm{H}_{\mathrm{LF}}(\text { odd })\left|\left(4 \mathrm{f}^{\mathrm{N}}\right) \Gamma\right\rangle}{\mathrm{E}(\Gamma)-\mathrm{E}(\mathrm{B} \beta)}+\frac{\left\langle\left(4 \mathrm{f}^{\mathrm{N}}\right) \Gamma^{\prime}\left|\mathrm{H}_{\mathrm{LF}}(\mathrm{odd})\right| \mathrm{B} \beta\right\rangle\left\langle\mathrm{B} \beta\left|\sum_{\mathrm{i}} \overrightarrow{\mathrm{r}}_{\mathrm{i}}\right|\left(4 \mathrm{f}^{\mathrm{N}}\right) \Gamma\right\rangle}{\mathrm{E}\left(\Gamma^{\prime}\right)-\mathrm{E}(\mathrm{B} \beta)}\right]$

An interesting estimation of the $4 \mathrm{f}-4 \mathrm{f}$ oscillator strengths can be made from equation (15). For an electric dipole allowed transition the oscillator strength can be as high as 1 . For rare earth ions the ligand field interaction is typically of the order of $100 \mathrm{~cm}^{-1}$ and the interconfigurational energy differences for the lowest excited configuration of opposite parity $\left(4 \mathrm{f}^{\mathrm{N}-1} 5 \mathrm{~d}\right)$ is typically of the order of $10^{5} \mathrm{~cm}^{-1}$. This gives a factor of $10^{-3}$ in equation (15), which squared leads to the typical order of magnitude of $4 \mathrm{f}-4 \mathrm{f}$ oscillator strengths $\left(10^{-6}\right)$.

The summation over $B$ and $\beta$ in equation (15) reminds the possibility of using a closure procedure, and indeed this is the next step in the Judd-Ofelt treatment. This summation becomes much more treatable if one assumes that the intraconfigurational energy differences are much smaller than the energy differences between the baricenters of the ground and excited configurations. Or, in other words, if one assumes that $\mathrm{E}(\Gamma)-\mathrm{E}(\mathrm{B} \beta) \cong \mathrm{E}\left(\Gamma^{\prime}\right)-\mathrm{E}(\mathrm{B} \beta) \cong \mathrm{E}_{\mathrm{b}}\left(4 \mathrm{f}^{\mathrm{N}}\right)$ $\mathrm{E}_{\mathrm{b}}(\mathrm{B})=\Delta \mathrm{E}(\mathrm{B})$. The main point now is to use the following relation involving two irreducible tensor operators $\mathrm{x}_{\mathrm{q}}{ }^{(\mathrm{k})}=\sum_{\mathrm{i}} \mathrm{X}_{\mathrm{q}}{ }^{(\mathrm{k})}$ (i) and $\mathrm{z}_{\mathrm{q}^{\prime}}{ }^{\left({ }^{\prime}\right)}=$ $\sum_{\mathrm{i}} \mathrm{z}_{\mathrm{q}^{\prime}}{ }^{\left(\mathrm{k}^{\prime}\right)}(\mathrm{i})^{7,8}$.

$\sum_{\beta}\left\langle\left(4 \mathrm{f}^{\mathrm{N}}\right) \Gamma^{\prime}\left|\mathrm{X}_{\mathrm{q}}^{(\mathrm{k})}\right| \mathrm{B} \beta\right\rangle\left\langle\mathrm{B} \beta\left|\mathrm{Z}_{\mathrm{q}^{\prime}}^{\left(\mathrm{k}^{\prime}\right)}\right|\left(4 \mathrm{f}^{\mathrm{N}}\right) \Gamma\right\rangle=$

$\sum_{\lambda, \mathrm{Q}}(-1)^{\mathrm{Q}}(2 \lambda+1)\left\langle 4 \mathrm{f}\left\|\mathrm{x}^{(\mathrm{k})}\right\| \mathrm{n} \ell\right\rangle\left\langle\mathrm{n} \ell\left\|\mathrm{z}^{\left(\mathrm{k}^{\prime}\right)}\right\| 4 \mathrm{f}\right\rangle\left(\begin{array}{ccc}\mathrm{k}^{\prime} & \mathrm{k} & \lambda \\ \mathrm{q}^{\prime} & \mathrm{q} & -\mathrm{Q}\end{array}\right)$

$\left\{\begin{array}{ccc}\mathrm{f} & \mathrm{k} & \ell \\ \mathrm{k}^{\prime} & \mathrm{f} & \lambda\end{array}\right\}\left\langle\left(4 \mathrm{f}^{\mathrm{N}}\right) \Gamma^{\prime}\left|\mathrm{U}_{\mathrm{Q}}^{(\lambda)}\right|\left(4 \mathrm{f}^{\mathrm{N}}\right) \Gamma\right\rangle$

In this equation the quantities in ( ) and \{\} are $3-j$ and $6-j$ symbols, respectively ${ }^{19-22}$. The monoelectronic reduced matrix elements involving $\mathrm{x}^{(\mathrm{k})}$ and $\mathrm{z}^{\left(\mathrm{k}^{\prime}\right)}$ contain the radial part corresponding to these operators, and $\mathbf{U}^{(\lambda)}$ is an irreducible unit tensor operator ${ }^{19-22}$. In the case of core excitations $(l=\mathrm{d}, \mathrm{n}=3$ and 4$)$ a minus sign appears in the right-hand-side of equation (16). In our case, the ranks $\mathrm{k}$ and $\mathrm{k}^{\prime}$ are equal to 1 (from the dipole operator) and $\mathrm{t}$ (from $\mathrm{H}_{\mathrm{LF}}(\mathrm{odd})$ ), respectively. Thus, it may be shown that the only difference between the two terms in the right-hand-side of equation (15) is in the 3-j symbols, which are related by

$\left(\begin{array}{ccc}1 & \mathrm{t} & \lambda \\ \mathrm{q} & \mathrm{p} & -\mathrm{Q}\end{array}\right)=(-1)^{1+\mathrm{t}+\lambda}\left(\begin{array}{ccc}\mathrm{t} & 1 & \lambda \\ \mathrm{p} & \mathrm{q} & -\mathrm{Q}\end{array}\right)$

Since $t$ is odd only even values of 1 will lead to nonvanishing values of $\vec{\mu}_{\text {ed }}$. From the triangularity rules for the $6-j$ symbol in equation (16), it follows that $\lambda \leq 2$ f, i.e. $\lambda \leq 6$. The unit tensor operator $\mathbf{U}^{(0)}$ is a scalar and cannot contribute to transition probabilities. Therefore, the operative values of $\lambda$ are 2, 4 and 6 .

The matrix element $\vec{\mu}_{\text {ed }}$ may then be put in the form:

$\vec{\mu}_{\text {ed }}=\sum_{\lambda, t, Q, p, q}(-1)^{Q}(2 \lambda+1)\left(\begin{array}{ccc}1 & t & \lambda \\ q & p & -Q\end{array}\right) B_{\lambda t p}^{\text {ed }}\left\langle\left(4 \mathrm{f}^{\mathrm{N}}\right) \Gamma^{\prime}\left|\mathrm{U}_{\mathrm{Q}}^{(\lambda)}\right|\left(4 \mathrm{f}^{\mathrm{N}}\right) \Gamma\right\rangle \overrightarrow{\mathrm{e}}_{\mathrm{q}} *$

where the spherical unit vectors satisfy the condition $\vec{e}_{q}{ }^{*} \cdot \vec{e}_{q^{\prime}}=\delta_{q q^{\prime}}$, and the quantities $B_{\lambda_{\mathrm{t} p}}^{\text {ed }}$ are given by:

$\mathrm{B}_{\lambda \mathrm{tp}}^{\mathrm{ed}}=\Xi(\mathrm{t}, \lambda) \gamma_{\mathrm{p}}^{\mathrm{t}}$

where
$\Xi(\mathrm{t}, \lambda)=2 \sum_{\mathrm{n}, \ell}\left\{\begin{array}{ccc}\mathrm{f} & 1 & \ell \\ \mathrm{t} & \mathrm{f} & \lambda\end{array}\right\}\left\langle\mathrm{f}\left\|\mathrm{C}^{(1)}\right\| \ell\right\rangle\left\langle\ell\left\|\mathrm{C}^{(\mathrm{t})}\right\| \mathrm{f}\right\rangle\langle 4 \mathrm{f}|\mathrm{r}| \mathrm{n} \ell\rangle\left\langle\mathrm{n} \ell\left|\mathrm{r}^{\mathrm{t}}\right| 4 \mathrm{f}\right\rangle \frac{1}{\Delta \mathrm{E}(\mathrm{n} \ell)}$

If one is not interested in the transition intensities between Stark levels $\left(\Gamma, \Gamma^{\prime}\right)$ but rather in the integrated intensities between $\mathrm{J}$ and $\mathrm{J}^{\prime}$ manifolds, to a first approximation the J-mixing effects may be neglected and the $4 \mathrm{f}^{\mathrm{N}}$ eigenstates in equation (17) may be replaced by the eigenstates in the intermediate coupling scheme defined in equation (3). Thus, the total electric dipole strength is a sum over $M_{J}$ and $\mathrm{M}_{\mathrm{J} \dot{c}}$ divided by $2 \mathrm{~J}+1$, assuming that the components of the initial $\mathrm{J}$ manifold are equally thermally populated. Using the Wigner-Eckart theorem and the orthogonality relation between $3-\mathrm{j}$ symbols, it may be easily shown that the total electric dipole strength in equation (13) is then given by:

$\mathrm{S}_{\text {ed }}=\frac{1}{2 \mathrm{~J}+1} \sum_{\lambda=2,4,6} \Omega_{\lambda}^{\mathrm{ed}}\left\langle\left(4 \mathrm{f}^{\mathrm{N}}\right) \psi^{\prime} \mathrm{J}^{\prime}\left\|\mathrm{U}^{(\lambda)}\right\|\left(4 \mathrm{f}^{\mathrm{N}}\right) \psi \mathrm{J}\right\rangle^{2}$

where

$\Omega_{\lambda}^{\text {ed }}=(2 \lambda+1) \sum_{\mathrm{t}, \mathrm{p}} \frac{\left|\mathrm{B}_{\lambda \mathrm{tp}}^{\mathrm{ed}}\right|^{2}}{2 \mathrm{t}+1}$

An alternative way of performing the summation in equation (15) has been the use of the average energy denominator method, introduced by Bebb and Gold ${ }^{28}$. The advantage is that one has to deal with a single average energy difference in equation (15). It has been shown that the predicted values of the so-called intensity parameters $B_{\lambda \text { tp }}^{\text {ed }}$ and $\Omega_{\lambda}^{\text {ed }}$ are very similar to those given by the standard Judd-Ofelt treatment ${ }^{14}$. The coefficient of spontaneous emission taking into account both the forced electric dipole and the magnetic dipole mechanisms, is then given by:

$A_{J J^{\prime}}=\frac{4 e^{2} \omega^{3}}{3 \hbar c^{3}}\left[\frac{n\left(n^{2}+2\right)^{2}}{9} S_{e d}+n^{3} S_{m d}\right]$

It should be noted, however, that the previous equation is valid as far as the $\mathrm{J}$-mixing is neglected. Otherwise a cross term between the electric dipole and magnetic dipole transition moments may appear. The corresponding expression for the oscillator strength may be obtained from the relation:

$P_{J^{\prime} J}=\frac{2 J+1}{2 J^{\prime}+1} \frac{m_{e} c^{3}}{2 \omega^{2} e^{2} n^{2}} A_{J J^{\prime}}$

\section{The dynamic coupling}

This mechanism was originally proposed by Jörgensen and Judd ${ }^{27}$ in an attempt to explain the uncommon intensity variation of certain 4f-4f transitions denominated hypersensitive transitions. A simplified visualization of this mechanism is shown in Figure 2.

The incident radiation field induces oscillating dipoles in the surrounding atoms and, as a consequence, an additional oscillating electric field is produced. This electric field, being produced close to the rare earth ion, has large local gradients and may induce $4 \mathrm{f}-4 \mathrm{f}$ transitions with oscillator strengths in the order of, or even greater than $10^{-6}$. To a first approximation the induced oscillating dipoles depend on the isotropic dipolar polarizabilities of the surrounding atoms, $\alpha$, as indicated in Figure 2. The interaction energy with the $4 \mathrm{f}$ electrons, $\mathrm{H}_{\mathrm{DC}}$, is given by:

$H_{D C}=e \sum_{i, j} \vec{\mu}_{j} \cdot \frac{\left(\vec{r}_{i}-\vec{R}_{j}\right)}{\left|\vec{r}_{i}-\vec{R}_{j}\right|^{3}}$ 


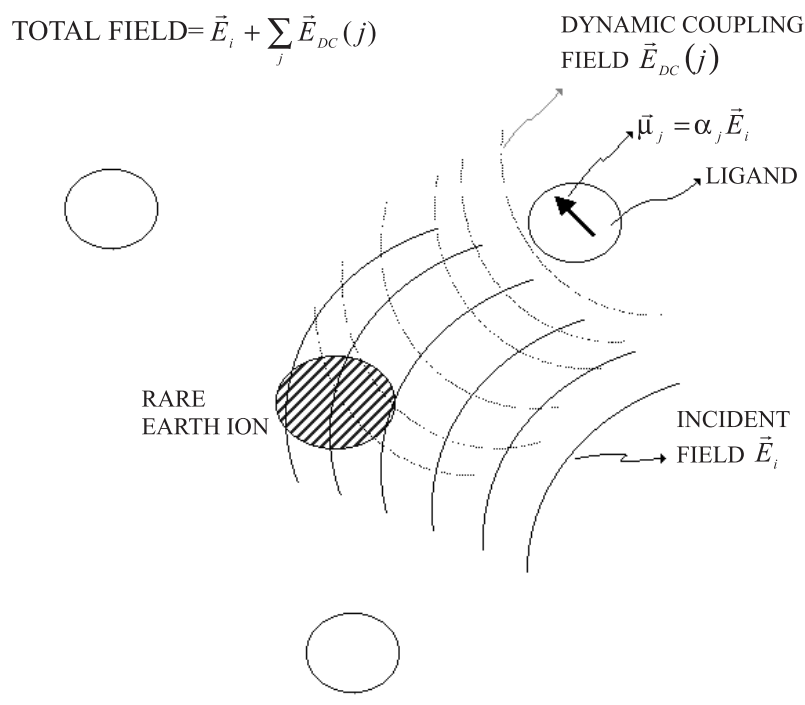

Figure 2. A pictorial representation of the dynamic coupling

$\mathrm{H}_{\mathrm{DC}}$ must be added to the interaction, with the incident field, that leads to the forced electric dipole mechanism. When expanded in terms of irreducible tensor operators ${ }^{19,20}$, the even rank components of $\mathrm{H}_{\mathrm{DC}}$ lead to a transition moment (in units of e) that has exactly the same form as the transition moment given in equation (17), that is:

$\vec{\mu}_{\mathrm{DC}}=\sum_{\lambda, \mathrm{t}, \mathrm{Q}, \mathrm{p}, \mathrm{q}}(-1)^{\mathrm{Q}}(2 \lambda+1)\left(\begin{array}{ccc}1 & \mathrm{t} & \lambda \\ \mathrm{q} & \mathrm{p} & -\mathrm{Q}\end{array}\right) \mathrm{B}_{\lambda t \mathrm{p}}^{\mathrm{DC}}\left\langle\left(4 \mathrm{f}^{\mathrm{N}}\right) \Gamma^{\prime}\left|\mathrm{U}_{\mathrm{Q}}^{(\lambda)}\right|\left(4 \mathrm{f}^{\mathrm{N}}\right) \Gamma\right\rangle \overrightarrow{\mathrm{e}}_{\mathrm{q}} *$

where

$\mathrm{B}_{\lambda \mathrm{tp}}^{\mathrm{DC}}=-\left[\frac{(\lambda+1)(2 \lambda+3)}{(2 \lambda+1)}\right]^{1 / 2}\left\langle 4 \mathrm{f}\left|\mathrm{r}^{\lambda}\right| 4 \mathrm{f}\right\rangle\left(1-\sigma_{\lambda}\right)\left\langle\mathrm{f}\left\|\mathrm{C}^{(\lambda)}\right\| \mathrm{f}\right\rangle \Gamma_{\mathrm{p}}^{\mathrm{t}} \delta_{\mathrm{t}, \lambda+1}$

and

$\Gamma_{p}^{t}=\left(\frac{4 \pi}{2 t+1}\right)^{1 / 2} \sum_{j} \frac{\alpha_{j}}{R_{j}^{t+1}} Y_{p}^{t^{*}}\left(\Omega_{j}\right)$

$\mathrm{Y}_{\mathrm{p}}^{\mathrm{t}}$ is a spherical harmonic and $\left(1-\sigma_{\lambda}\right)$ in equation (26) is a shielding factor, due to the filled $5 \mathrm{~s}$ and $5 \mathrm{p}$ sub-shells of the rare earth ion ${ }^{14}$. The total intensity parameter is now $B_{\lambda t p}=B_{\lambda t p}^{\text {ed }}+B_{\lambda t p}^{D C}$, which is the quantity to be used in equation (21) to obtain the total $\Omega_{\lambda}$ parameters.

Several interesting aspects may be discussed about the forced electric dipole and dynamic coupling mechanisms. An analysis from typical values of the quantities that appear in equations (18) and (26) indicate that these two mechanisms contribute to the total transition moment with opposite signs ${ }^{16}$. Both the odd rank ligand field parameters, $\gamma_{\mathrm{p}}^{\mathrm{t}}$, and the polarizability dependent quantities $\Gamma_{\mathrm{p}}^{\mathrm{t}}$ contain generally the same type of sum over the surrounding atoms. Therefore, they carry out the same symmetry information. The only difference is that $\Gamma_{\mathrm{p}}^{\mathrm{t}}$ does not depend on the spherical harmonic of rank $1\left(\mathrm{Y}_{\mathrm{p}}^{1}\right)$ as it may be noted from the Kronecker's delta in equations (26).

As the site occupied by the rare earth ion becomes more symmetric, the lower rank $\gamma_{\mathrm{p}}^{\mathrm{t}}$ and $\Gamma_{\mathrm{p}}^{\mathrm{t}}$ tend to vanish more rapidly than the higher rank ones, or in a more general way, the former quantities are more sensitive to changes in symmetry than the latter, though the higher rank $\gamma_{\mathrm{p}}^{\mathrm{t}}$ and $\Gamma_{\mathrm{p}}^{\mathrm{t}}$ are more sensitive to changes in distances. This goes in the correct sense towards the rationalization of the so-called hypersensitive transitions, which are normally dominated by the effective operator $\Omega_{2} \mathbf{U}^{(2)}$. On the other hand, this also seems to agree with the empirical suggestion that the effects involving slight changes in the positions of the ligands belonging to the second coordination shell could be reflected in the $\Omega_{4} \mathbf{U}^{(4)}$ and $\Omega_{6} \mathbf{U}^{(6)}$ effective operators $^{29,30,31}$. However, it has been observed that the symmetry alone cannot account for the enormous variation sometimes observed in the intensities of those hypersensitive transitions for different chemical environments. Theoretical estimates have shown that the dynamic coupling contribution is able to account for this enormous intensity variation through the polarizabilities of the surrounding atoms, or groups of atoms.

Thus, for example, in going from the gaseous compound $\mathrm{NdF}_{3}$ to gaseous $\mathrm{NdI}_{3}$ there is a change in polarizability, from the ion $\mathrm{F}^{-}$to the ion $\mathrm{I}^{-}$, of almost one order of magnitude. This might produce a change of almost two orders of magnitude in the intensities dominated by $\Omega_{2} \mathbf{U}^{(2)}$. Abnormal changes in the intensities dominated by $\Omega_{4} \mathbf{U}^{(4)}$ and $\mathrm{W}_{6} \mathbf{U}^{(6)}$ may not occur, since for these cases the remarkable increase in the distance $\mathrm{Nd}-\mathrm{X}(\mathrm{X}=\mathrm{F}$ and $\mathrm{I})$ may compensate for the increase in the polarizability values. This can explain the fact that nearly all glass materials containing trivalent rare earth ions (and also the aqua ions and other complexes in solution) show a moderate variation of both $\Omega_{4}$ and $\Omega_{6}$ parameters, between 1 and $5 \times 10^{-20} \mathrm{~cm}^{21,32}$, contrarily to the changes in the $\Omega_{2}$ values, which vary from less than 1 to $40 \times 10^{-20} \mathrm{~cm}^{2}$ in condensed matter (table 1 of Ref. 1) and even up to $275 \times 10^{-20} \mathrm{~cm}^{2}$ for $\mathrm{NdI}_{3}$ vapor.

A point that should be stressed here is that, in contrast with a common procedure found in the literature in the case of rare earthdoped glasses, the dynamic coupling mechanism cannot, in any circumstances, be neglected. When the $\Omega_{\lambda}$ intensity parameters are phenomenologically determined from experimental intensities, the forced electric dipole and dynamic coupling mechanisms are absorbed simultaneously and cannot be distinguished. Therefore, when treating energy transfer processes between rare earth ions, one should bear in mind that in the dipole-dipole or dipole- quadrupole expressions for the transfer rates the $\Omega_{\lambda}$ 's which appear refer only to the forced electric dipole contribution, that is, $\Omega_{\lambda}^{\text {ed. }}$. This is one of the reasons that motivate the theoretical calculations of the individual $B_{\lambda t p}^{\text {ed }}$ and $B_{\lambda t p}^{D C}$ contributions. These theoretical calculations in vitreous materials turn out to be an enormous problem, due to the large variety of different site symmetries occupied by the rare earth ion, unless a model system with a well defined statistical distribution of site symmetry types is available. In this case the $\Omega_{\lambda}$ parameters represent average values over all types of sites.

\section{INTENSITY PARAMETERS IN VITREOUS MATERIALS}

One of the effects of a distribution of different symmetry sites occupied by the rare earth ion is to produce the inhomogeneous line broadening. The Stark levels overlap in such a way that, in most cases, even the fluorescence line-narrowing technique cannot help to identify a particular site occupied by the rare earth ion. Figure 3 shows the emission spectrum of the $\mathrm{Eu}^{3+}$ ion in fluoroborate glasses ${ }^{33}$, where this effect can be clearly noted, particularly in the ${ }^{5} \mathrm{D}_{0} \rightarrow{ }^{7} \mathrm{~F}_{2}$ hypersensitive transition at $\sim 612 \mathrm{~nm}$.

As already mentioned, the $\Omega_{\lambda}$ intensity parameters in vitreous materials correspond to statistical average values over all sites, and these values, determined experimentally, incorporate both the forced electric dipole and the dynamic coupling contributions. The dependence on the polarizabilities of the neighboring ions confers to the latter mechanism a stronger dependence on the nature of the chemical environment. For the sake of comparison, in a $100 \%$ ionic model of the ligand field interaction, the charge of the fluorine and chlorine ions, for example, is -1 , in units of the electronic charge, while their dipolar isotropic polarizabilities are, respectively, $1 \AA^{3}$ and $\sim 3 \AA^{3}$. This may account for the hypersensitive behavior of certain $4 \mathrm{f}-4 \mathrm{f}$ transitions, which are generally dominated by $\Omega_{2} \mathbf{U}^{(2)}$. 


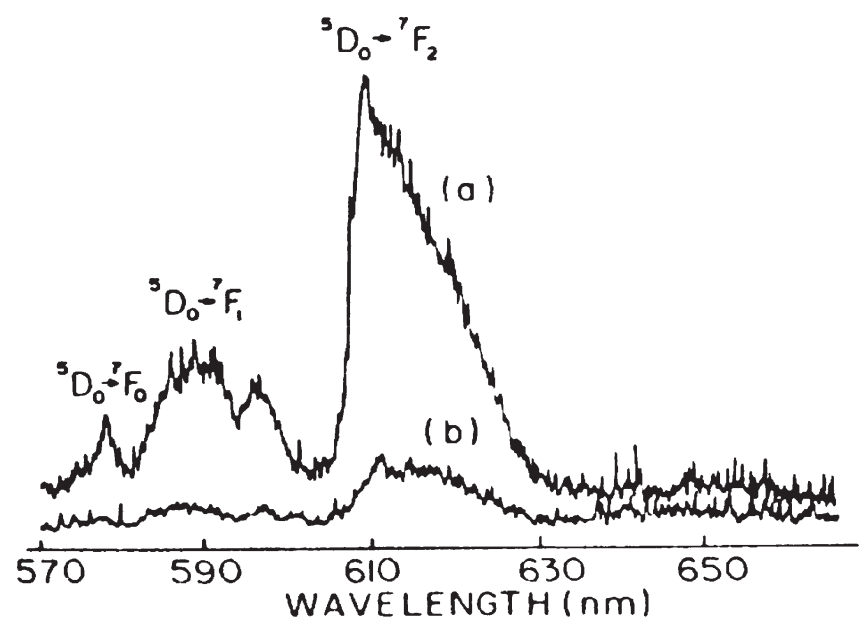

Figure 3. Luminescence spectrum of the $E u^{3+}$ ion, in the presence (a) and in the absence $(b)$ of silver particles, in a fluoroborate glass

A correlation has been noticed in the sense that compounds expected to have a higher degree of covalency tend to present higher values of $\Omega_{2}{ }^{1}$, suggesting that in these cases the dynamic coupling mechanism dominates. This correlation can be clearly seen from the $\Omega_{2}$ values collected in table 1 of Ref. 1 and in Ref. 32, where the oxides and chalkogenides present higher values for this intensity parameter than the fluorides, which are known to have the least covalent bonding with the rare earths.

The case of the fluoride phosphate $\mathrm{Eu}^{3+}$ and $\mathrm{Tb}^{3+}$ glasses, for instance, is another example that nicely illustrates this correlation. In these glasses, $\Omega_{2}$ increases linearly with the increase of the phosphate content, due to the replacement of fluoride ions presenting lower electron polarizability by the oxygen atoms having higher polarizability ${ }^{34}$. Another correlation has also been noted between the $\Omega_{4}$ and $\Omega_{6}$ parameters and the amplitudes of localized vibrational modes involving the rare earth ion $^{1}$, giving an indication of the rigidity of the material. It is, however, rather difficult to rationalize this correlation in terms of the quantities that appear in equations (18) and (26).

An interesting and controversial case of intensity parameters is the $\mathrm{Pr}^{3+}$ ion. In many compounds with $\mathrm{Pr}^{3+}$ it is found that the phenomenological $\Omega_{2}$ parameter is negative, what, from the definition of the $\Omega_{\lambda}$ [equation (21)], is not acceptable. It has been argued ${ }^{9}$ that for this ion the lowest excited configuration of opposite parity, $4 \mathrm{f} 5 \mathrm{~d}$, is too close $\left(\sim 50000 \mathrm{~cm}^{-1}\right)$ to the ground configuration $\left(4 \mathrm{f}^{2}\right)$, invalidating the approximation made on the energy denominators in equation (15).

There are different ways in which corrections could be introduced. One is to take into account the $4 \mathrm{f}^{\mathrm{N}}$ wavefunctions up to higher than first order in perturbation theory ${ }^{12}$. Another one is, for example, to make appropriate expansions on the inverse of the energy differences in equation (15), as it has been done in Ref. 35. In either way one finds that the odd rank effective operators $\Omega_{\lambda}{ }^{\text {ed }} \mathbf{U}^{(\lambda)}(\lambda=1,3$ and 5) may be of significance when $\operatorname{DE}(5 \mathrm{~d})$ is small, as in the case of the $\mathrm{Pr}^{3+}$ ion. However, one should bear in mind that even in this case, depending on the chemical environment, the dynamic coupling mechanism may dominate, what would make more difficult to evaluate precisely the effect of the odd rank effective operators. There are evidences in the case of the isoelectronic ion $\mathrm{U}^{4+}\left(5 \mathrm{f}^{2}\right)$, found by Auzel $^{36}$, indicating a rather independent behavior of the $5 \mathrm{f}-5 \mathrm{f}$ transition intensities with the position of the $5 \mathrm{f} 6 \mathrm{~d}$ excited levels of opposite parity, suggesting a dominance of the dynamic coupling mechanism.
Another aspect about $\operatorname{Pr}^{3+}$ compounds concerns the statistical procedure, which is usually adopted for extracting the $\Omega_{\lambda}$ intensity parameters from experimental oscillator strengths (least-squares method). It is possible that in this case the set of linear equations is particularly sensitive to very small variations, within experimental errors, in the oscillator strengths. A method in which branching ratios are included in the least-squares procedure was proposed by Quimby and Miniscalco ${ }^{37}$, and a method in which the standard deviation for each individual oscillator strength is introduced in the minimization procedure has been used by Goldner and Auzel ${ }^{38}$, both leading to reliable intensity parameters.

\section{CONCLUDING REMARKS}

Some very basic aspects of the theory of $4 \mathrm{f}-4 \mathrm{f}$ transition intensities applied to vitreous materials have been discussed above. The characteristics of the intra-atomic rare earth free ion and ligand field interactions, as well as the formalisms of the forced electric dipole and dynamic coupling mechanisms of $4 \mathrm{f}-4 \mathrm{f}$ intensities, have been outlined. One of the main points was to call attention to the contribution from the dynamic coupling mechanism to the intensities, a point that has been commonly overlooked in the literature of rare earth doped glass materials. Not taking into account this mechanism is equivalent to assume that the phenomenological $\Omega_{\lambda}$ intensity parameters coincide with $\Omega_{\lambda}{ }^{\text {ed }}$, corresponding to the forced electric dipole contribution alone. This would be a clear misinterpretation of the theory. From the theoretical expressions given in equations (18) and (26) it is possible to rationalize the correlation between $\Omega_{2}$ and covalence, as discussed in Ref. 1. However, the same is not evident concerning the correlation between $\Omega_{4}$ and $\Omega_{6}$ and the rigidity of the medium. The case of the $\operatorname{Pr}^{3+}$ ion has been briefly discussed under the light of the forced electric dipole and dynamic coupling mechanisms, and attention has been called to the fact that for this ion statistical problems may arise when determining phenomenological intensity parameters from experimental oscillator strengths.

\section{ACKNOWLEDGMENTS}

Financial support from the CNPq (Brazilian agency), RENAMI and IMMC (Brazilian projects), and the FCT (Portuguese agency, POCTI CTM/33653/00 program) is acknowledged.

\section{REFERENCES}

1. Reisfeld, R.; Jörgensen, C. K. In Handbook on the Physics and Chemistry of Rare Earths; Gschneidner Jr., K. A.; Eyring, L., eds.; North-Holland: Amsterdam, 1987, vol. 9, ch. 58, p. 1.

2. Rare Earth Doped Fiber Lasers and Amplifiers; Digonnet, M. J. F., ed.; Marcel Dekker: New York, 1993.

3. Fuxi, G.; Optical and Spectroscopic Properties of Glasses, Springer-Verlag: Berlin, 1992.

4. Weber, M. J. In ref. 1, vol. 4, ch. 35, p. 275.

5. Collins, S. F.; Baxter, G. W.; Wade, S. A.; Sun, T.; Grattan, K. T. V.; Zhang, Z. Y.; Palmer, A. M.; J. Appl. Phys. 1998, 84, 4649.

6. Downing, E.; Hesselink, L.; Ralston, J.; MacFarlane, R.; Science 1996, 273, 1185 .

7. Judd, B. R.; Phys. Rev. 1962, 127, 750.

8. Ofelt, G. S.; J. Chem. Phys. 1962, 37, 511

9. Peacock, R. D.; Structure and Bonding 1975, 22, 83.

10. Judd, B. R.; J. Chem. Phys. 1979, 70, 4830.

11. Reid, M. F.; Dallara, J. J.; Richardson, F. S.; J. Chem. Phys. 1983, 79, 5743.

12. Smentek-Mielczarek, L.; Hess Jr., B. A.; J. Chem. Phys. 1987, 87, 3509.

13. Reid, M. F.; Betty, N. G.; Mol. Phys. 1989, 67, 407.

14. Malta, O. L.; Ribeiro, S. J. L.; Faucher, M.; Porcher, P.; J. Phys. Chem. Solids 1991, 52, 587.

15. Orlovskii, Y. V.; Pukhov, K. K.; Basiev, T. T.; Tsuboi, T.; Optical Materials 1995, 4, 583 . 
16. Malta, O. L.; Couto dos Santos, M. A.; Thompson, L. C.; Ito, N. K.; J. Lumin. 1996, 69, 77.

17. Basiev, T. T.; Orlovskii, Y. V.; Pukhov, K. K.; Sigachev, V. B.; Doroshenko, M. E.; Vorob'ev, I. N.; J. Lumin. 1996, 68, 241.

18. Racah, G.; Phys. Rev. 1949, 76, 1352.

19. Judd, B. R.; Operator Techniques in Atomic Spectroscopy, McGraw-Hill Book Company: New York, 1963.

20. Wybourne, B. G.; Spectroscopic Properties of Rare Earths, WileyIntersciences: New York, 1965.

21. Silver, B. L.; Irreducible Tensor Methods: An Introduction for Chemists, Academic Press: London, 1976.

22. Condon, E. U.; Odabasi, H.; Atomic Structure, Cambridge University Press, 1980

23. Prather, J. L.; Atomic Energy Levels in Crystals, National Bureau of Standards Monograph 19, NBS: Washington, 1961.

24. Görller-Walrand, C.; Binnemans, K. In ref. 1, vol. 23, ch. 155, p. 121.

25. Broer, L. J. F.; Gorter, C. J.; Hoogschagen, J.; Physica 1945, 11, 231.

26. Deutschbein, O.; Ann. Physik 1939, 36,183.
27. Jörgensen, C. K.; Judd, B. R.; Mol. Phys. 1964, 8, 281.

28. Bebb, H. B.; Gold, A.; Phys. Rev. 1966, 143, 1.

29. Brito, H. F.; Malta, O. L.; Souza, L. R.; Meneses, J. F. S.; Carvalho, C. A. A.; J. Non-Cryst. Solids 1999, 247, 129.

30. Bizeto, M. A.; Constantino, V. R. L.; Brito, H. F.; J. Alloys Compd. 2000, $311,159$.

31. Carlos, L. D.; Messaddeq, Y.; Brito, H. F.; Sá Ferreira, R. A.; de Zea Bermudez, V.; Ribeiro, S. J. L.; Adv. Mater. 2000, 12, 594.

32. Görller-Walrand, C.; Binnemans, K. In ref. 1, vol. 25, ch. 167, p. 101.

33. Malta, O. L.; Santa-Cruz, P. A.; de Sá, G. F.; Auzel, F.; J. Lumin. 1985, $33,261$.

34. Ebendorff-Heidepriem, H.; Ehrt, D.; J. Non-Cryst. Solids 1996, 208, 205.

35. Flórez, A.; Malta, O. L.; Messaddeq, Y.; Aegerter, M.; J. Non-Cryst. Solids 1997, 213/214, 315 .

36. Auzel, F.; personal communication.

37. Quimby, R. S.; Miniscalco, W. J.; J. Appl. Phys. 1994, 75, 613.

38. Goldner, P.; Auzel, F.; J. Appl. Phys. 1996, 79, 7972. 